\title{
Determination of Combining Ability for Some New Yellow Maize Inbred Lines Using Line X Tester Model
}

\author{
REHAB A. BAYOUMI, S.M. Shoker, G.Y. Hamam and A.A.A. EL-Hosary \\ Department of Agronomy, Faculty of Agriculture, Benha University, Egypt.
}

\begin{abstract}
This investigation was carried out to study the combining ability effects of 18 parents of maize (15 inbred lines and 3 testers) crossed in line $\mathrm{x}$ tester scheme for phonological, plant and yield characteristics. A yield trial included the 45 top crosses, 15 inbred lines, 3 testers along with the two check hybrids S.C. 168 and TWC 352. The experiment was conducted at two planting dates on $15^{\text {th }}$ May and $15^{\text {th }}$ June at the Agric. Res. and Experimental Station of the Fac. of Agric., Moshtohor Benha University. The randomized complete blocks design with three replications was used in this study. Significant mean squares due to crosses (C), inbred lines (L), testers (T) and line $\mathrm{x}$ tester (LxT) were found for most studied traits of both and across sowing dates. Variance for C, L, T and LxT with sowing dates interaction on the most studied traits was significance. $\delta^{2}$ sca played the major role in determining the inheritance of all traits. The magnitude of the interaction of $\delta^{2}$ SCA $x$ sowing date (SD) was generally higher than for $\delta^{2}$ GCA X SD. This finding indicates that non-additive is more affected by SD than additive and additive $\mathrm{x}$ additive. Three crosses exhibited significant superiority over high check hybrid S.C. G 168. The best top crosses were $T_{3} \times L_{2}$ and $T_{3} \times L_{15}$ at both and across sowing dates. Also, ten top crosses at combined analysis did not differ significantly than the best check hybrid for the mention trait. L2, L7, L14 and L15 were good general combiners for grain yield plant ${ }^{-1}$ and L9, L10 and L13 for earliness. The top-cross T1 with each of inbred lines 2 , 5 and 6, T2 with each of L1 and L12 and T3 with each of L3,L4 and L12 for earliness; T1xL11, T1xL12, T1xL14, T2xL3, T2xL12 and T3xL2 for grain yield plant ${ }^{-1}$ gave the best $\hat{S}_{i j}$ effects.
\end{abstract}

Key words: Maize, Inbred lines, Testers, Combining ability. GCA and SCA.

\section{Introduction}

Corn (Zea mays L.) is used as human food, poultry and livestock feeding, green fodder and silage for animal feeding. Moreover, it is also used for industrial purposes such as manufacturing starch and cooking oils. Maize is one of the most important cereal crops that plays a great role in narrowing the gap between production and consumption of grains in Egypt through increasing its cultivated area and enhancement of its productivity per unit area. In 2016 the area grown by this crop in Egypt was 0.75 Million hectares (1.76 million feddans) with an annual grain production of 6 Million metric tons and an average productivity of $8 \mathrm{Mg} \mathrm{ha}^{-1}$ (23.8 ardabs/feddan). (One feddan; fed $=4200 \mathrm{~m}^{2}$ and one ardab; $\operatorname{ard}=140 \mathrm{Kg}$ ). (USDA 2018).

Evaluating inbred lines is of prime importance for hybrid production. Therefore, it is important to know nature and number of tester parents to be used for evaluating inbred lines. The top crosses test with a broad and narrow base testers is the most common procedure for the evaluating process. Nature and number of testers to be used in the line $\mathrm{x}$ tester model for evaluating inbred lines is still unsolved problem. In this regard, the choice of a suitable tester is an important decision. Matzinger (1953) showed that a narrow genetic base tester contributes more to line $\mathrm{x}$ tester interaction than does a heterogeneous one. Davis (1927), Jenkins (1935) and Sprague (1939) suggested the method of early testing that is greatly affected by the nature and number of testers needed for efficient evaluation of inbred lines. Sprague and Tatum (1942) was the first to partition the total combining ability effects of the lines into general (GCA) and specific (SCA) combining ability. The suitable tester should maximize information on evaluating inbred lines for combining ability. The two main genetic parameters GCA and SCA are essential in developing breeding strategies. Furthermore, the magnitude of genetic components for a certain trait would depend mainly upon the environmental fluctuations under which the breeding populations will be tested. Therefore, much effort has been devoted by corn breeders to estimate the interactions between genetic components and environments.

The objectives of this study were to (1) evaluate some inbred lines of maize., (2) provide information of suitable testers for testing of inbred lines and (3) determine GCA and SCA as well as relative superiority relative to check hybrids involved in the manifestation of grain yield and other agronomic traits.

\section{Materials And Methods}

Fifteen new inbred lines in $\mathrm{S}_{7}$ of yellow maize were top crossed to three different testers in 2016 summer season. The female inbred lines were M 801 (L1), M 802 (L2), M 803 (L3), M 804 (L4), M 805 (L5), M 806 (L6), M 807 (L7), M 808 (L8), M 809 (L9), M 810 (L10), M 811 (L11), M 812 (L12), M 813 (L13), M 814 (L14) and M 815 (L15). The three testers were the population yellow Sahka Pop. (T1), 
the single cross M Y 10 (T2) and the yellow inbred line M L 212 (T3). Except for Sakha Population which was obtained from ARC, Egypt all plant materials were developed at the Department of Agronomy, Faculty of Agriculture, Benha University, Egypt by Prof. Dr. Ali El-Hosary.

The current experiment was carried out in two succeeded seasons at Experiment and Research Station of Moshtohor, Benha University, Kalubia Governorate, Egypt. In the first summer season 2016, top crosses were constituted. The fifteen yellow inbred lines and the three testers were sown on $25^{\text {th }}$ May and $3^{\text {th }}$ June in order to overcome the differences in flowering time and to secure enough hybrid seeds. In the second summer season 2017, two adjacent experiments were conducted on two sowing dates i.e. $15^{\text {th }}$ May and $15^{\text {th }}$ June. In each experiment, included the 45 top crosses, 15 inbred lines, 3 testers along with two check cultivars single cross S.C. G.168 and T.W.C. G.352 from ARC. The experimental design was randomized complete blocks design with three replications. Each plot consisted of one ridge of $8 \mathrm{~m}$ long and $70 \mathrm{~cm}$ width. Each hill was spaced $25 \mathrm{~cm}$ apart with two kernels planted per hill and later thinned to one plant per hill. The plots were irrigated immediatty after sowing and the second irrigation was given after 21 days from sowing. The plants were then irrigated at intervals of 10-15 days. The plots were informally fertilized at the rate of $120 \mathrm{~kg}$ of nitrogen per feddan $\left(1 \mathrm{feddan}=4200 \mathrm{~m}^{2}\right)$ given before the first and second irrigations. The other cultural practices of maize growing were properly practiced.

Data for the following traits were recorded on 15 individual guarded plants chosen at random from each plot, except for days to $50 \%$ tasseling and silking where the main plot basis was used. Data included days to $50 \%$ tasseling, days to $50 \%$ silking, plant and ear heights (cm), No. of rows ear-1 ${ }^{-1}$ No. of kernels row ${ }^{-}$ ${ }^{1}, 100$-kernel weight $(\mathrm{g})$ and grain yield plant ${ }^{-1}(\mathrm{~g})$.

Analysis of variance was performed for each sowing date as well as for combined data after homogeneity test across sowing dates according to Steel and Torrie (1980). Combining ability analysis of line $\mathrm{x}$ tester was conducted based on the procedure developed by Kempthorne (1957).

\section{Results and Discussion}

Table (1) reveals that sowing date mean squares were significant for all traits, indicating over all differences between the two sowing dates, with mean values in early sowing being higher than those in late sowing for all traits (Table 1). The increase in these traits at early sowing date may be due to the prevailing favorable temperature and day length leading to greater vegetative growth, yield and its components of corn plants. Therefore, the first sowing date seemed to be non-stress environment. These results are in agreement with those obtained by Hani et al (2006), Hefny and Aly (2008), Ngaboyisonga et al (2009),
Tamilarasi and Vetriventhan (2009), EL-Badawy et al (2010) and Abd El-Aal (2012). The present results confirm the earliar view of Kang (1998) who mentioned a prominent role of environment on phenotypic expression of agronomic traits. Bello and Olaoye (2009) suggested that variation in climate (rainfall, sunshine, relative humidity, etc.) could be an important factor in breeding for desirable traits including grain yield. Earliness in maize is favorable for escaping destructive injuries caused by Sesamia cretica, Chilo simplex and Pyrausta nubilialis. Similar results were reported by El-Hosary and El-Badawy (2005), El-Hosary et al (2006), El-Hosary and Elgammaal (2013), and El-Hosary (2014) .

Crosses mean squares were significant for all the studied traits at both sowing dates as well as the combined analysis, indicating the wide diversity among the parental materials used in the present study. Significant crosses $\mathrm{x}$ sowing date mean squares were obtained for all traits except number of rows / ear, number of kernels/ row and 100-kernel, revealing that the tested crosses varied from each other and ranked differently from sowing date to another.

Line mean squares were significant for all traits at early, late sowing dates and across environment, indicating the wide diversity among those inbred lines. Significant lines $\mathrm{x}$ sowing date mean squares were detected for all traits except number of rows/ ear, number of kernels/ row and 100-kernel weight. These finding indicate that parental inbred lines differ in their mean performance in most traits.

Significant mean squares due to testers were obtained for all traits in both sowing dates as well as the combined analysis except No of rows/ ear at early sowing date, No of kernels/ row at late sowing date as well as the combined analysis. Such results indicated a wide range of variability among parental testers. In addition, tester mean squares were much higher than those of lines for five traits of studied traits. Such results revealed that testers contributed much more to the total variation as compared to inbred lines. Also, the interaction between tester $\mathrm{x}$ sowing date mean squares were significant for all traits except, plant height. This indicated that the testers behaved somewhat differently from one sowing date to another.

Significant line $\mathrm{x}$ tester mean squares were obtained for all traits except plant height at early sowing date as well as the combined data, ear diameter and 100-kernel weight at early sowing date.

Significant interaction between line by tester $\mathrm{x}$ sowing date mean squares were obtained for all traits except, plant height, number of kernel/ row, 100kernel weight.

The estimates of variances due to GCA, SCA and their interactions with sowing dates (Table 1) showed that $\delta^{2}$ SCA played the major role in determining the inheritance of most studied traits, revealing that the largest part of the total genetic variability associated with these traits was a result of non- additive gene 
action. These results for most studied traits support the findings of El-Hosary (1985), Sofi and Rather (2006) and Basbag et al (2007), who reported that $\delta^{2}$ SCA was important in the inheritance of grain yield plant $^{-1}$ and other agronomic traits. The magnitude of the interactions for $\delta^{2}$ SCA $x$ sowing date $(\mathrm{SD})$ was generally higher than for $\delta^{2}{ }_{\mathrm{GCA}} \times \mathrm{SD}$. This finding indicates non-additive type of gene action to be more affected by sowing date (SD) than additive and additive $\mathrm{x}$ additive types of gene action. This is in agreement with the findings of several investigators who reported that $\delta^{2} \mathrm{SCA}$ is more sensitive to environmental changes than $\delta^{2}{ }_{\mathrm{GCA}}$ (Gilbert 1958 and El-Hosary (2014)).

Means of the 45 top crosses, and three testers in the combined analysis for all studied traits are presented in Table 2. Most studied top crosses recorded lower values for the measured traits at delayed sowing.

For tasseling date eleven top crosses was significantly lower than the best earlier check variety at the combined analysis. The top crosses $\mathrm{T}_{1} \times \mathrm{L}_{9}$, $\mathrm{T}_{2} \mathrm{XL}_{15}, \mathrm{~T}_{2} \mathrm{XL}_{13}$ and $\mathrm{T}_{3} \mathrm{xL}_{10}$ gave the lowest mean values for tasseling date at the combined data.

For silking date, five crosses exhibited significantly earliness than the earlier check variety $(\mathrm{T}$ W 352). The top crosses $\mathrm{T}_{3} \mathrm{xL}_{4}, \mathrm{~T}_{1} \mathrm{xL}_{3}, \mathrm{~T}_{1} \mathrm{xL}_{4}, \mathrm{~T}_{1} \mathrm{xL}_{11}$, $\mathrm{T}_{1} \mathrm{XL}_{12}$ and $\mathrm{T}_{2} \mathrm{XL}_{13}$ gave the lowest mean values for this trait at the combined data.

Regarding plant height three top crosses expressed significant lowest values as compared with the best check variety T.W. G 352. The top crosses $\mathrm{T}_{1} \mathrm{XL}_{1}, \mathrm{~T}_{1} \mathrm{xL}_{2}$ and $\mathrm{T}_{1} \mathrm{xL}_{13}$ were the best among the studied crosses since they expressed the lowest significant values of this trait.

As for ear height, four top crosses showed significant lowest values as compared to best check variety from the two check hybrids. The top crosses $\mathrm{T}_{1} \mathrm{XL}_{12}, \mathrm{~T}_{1} \mathrm{xL}_{1}, \mathrm{~T}_{1} \mathrm{xL}_{2}, \mathrm{~T}_{1} \mathrm{xL}_{6}, \mathrm{~T}_{1} \mathrm{xL}_{7}, \mathrm{~T}_{1} \mathrm{xL}_{8}, \mathrm{~T}_{1} \mathrm{xL}_{2}$, $\mathrm{T}_{1} \mathrm{XL}_{13}$ and $\mathrm{T}_{2} \mathrm{xL}_{6}$ at the combined analysis which had the best values for plant height showed also the most desirable values for ear height. Therefore, these top crosses are prospective in maize breeding program.

For number of rows/ ear, the top crosses $\mathrm{T}_{1} \mathrm{xL}_{12}$, $\mathrm{T}_{2} \times \mathrm{L}_{15}$ and $\mathrm{T}_{3} \times \mathrm{L}_{2}$ exhibited significant higher than the best check hybrid.

For number of kernels/row, twenty four of the top crosses significantly surpassed the best check hybrid $\mathrm{G}$ 168. However, the crosses $\mathrm{T}_{2} \times \mathrm{L}_{7}, \mathrm{~T}_{2} \mathrm{XL}_{10}, \mathrm{~T}_{3} \mathrm{XL}_{1}$, $\mathrm{T}_{3} \mathrm{XL}_{2}, \mathrm{~T}_{3} \mathrm{xL}_{4}, \mathrm{~T}_{3} \mathrm{xL}_{5}, \mathrm{~T}_{3} \mathrm{xL}_{7}, \mathrm{~T}_{3} \mathrm{xL}_{9}, \mathrm{~T}_{3} \mathrm{xL}_{10}, \mathrm{~T}_{3} \mathrm{xL}_{11}$, and $\mathrm{T}_{3} \times L_{15}$, surpassed the best check hybrids.

Regarding grain yield/ plant, the highest check mean values were recorded by S.C. G 168 . However, three crosses exhibited significant superiority over this check hybrid.

The best top crosses were $\mathrm{T}_{3} \mathrm{XL}_{2}$ and $\mathrm{T}_{3} \mathrm{XL}_{15}$ at both and across sowing dates. Also, ten top crosses at combined analysis did not differ significantly than the best check hybrid.
From such results it could be concluded that the previous top crosses could be efficient and prospective in maize breeding programs since they expressed significant desirable effects for grain yield and for one or more of yield components.

The fluctuations of hybrid performance from sowing date to another were detected for most traits. These results would be due to significance of the interaction between hybrids and sowing date.

General combining ability effects for parents across sowing dates are presented in Table (3). There is no specific line recorded a desirable $\left(\hat{g}_{i}\right)$ effects for all traits. Desirable and significant $\left(\hat{g}_{i}\right)$ effects were obtained by L1, L2, L12 and L13 for short plant and low ear height, and L9, L10 and L13 for earliness. As expected, the tester L M 212 (T3) which had a broad genetic base, gave highly significant desirable $\left(\hat{g}_{i}\right)$ effects for most studied traits. Moreover, H 102, (T2) was not a good combiner for most studied traits.

This observation further confirms the previous findings and experience of many maize breeders. L2, L12 and L15 were the best general combiners for No of rows ear ${ }^{-1}$, L7, L13, 14 and L15 for No of kernels row $^{-1}, \mathrm{~L} 5, \mathrm{~L} 7$ and L10 for 100-kernel weight and L2, L7, L14 and L15 for high grain yield plant ${ }^{-1}$ in both and across sowing dates.

The superiority of inbred lines as good testers were noticed by several investigators Al-Naggar et al (1997), Amer (2002), Ibrahim and Ghonemy (2010) and El-Hosary (2014). These results indicated that these parental inbred lines possess favorable genes and that improvement in yield may be attained if they are used in a hybridization program.

Specific combining ability effects of the top crosses at combined across the two sowing dates are presented in Table (4).

The greatest inter- and intra-allelic interactions as deduced from $\hat{S}_{i j}$ effect were observed by top-crosses between T1 with each of L2, L5 and L6, T2 with each of L1 and L12 and T3 with each of L3,L4 and L12 for earliness (tassling and silking); T2xL8 for plant height; $\mathrm{T}_{3} \mathrm{XL}_{11}, \mathrm{~T}_{3} \mathrm{XL}_{13}, \mathrm{~T}_{3} \mathrm{XL}_{14}$ and $\mathrm{T}_{3} \mathrm{XL}_{15}$ for ear height; $\mathrm{T}_{3} \mathrm{x} \mathrm{L}_{2}$ For number of rows/ ear; $\mathrm{T}_{1} \mathrm{xL}_{8}, \mathrm{~T}_{2} \mathrm{XL}_{3}$, $\mathrm{T}_{2} \times \mathrm{L}_{10}$ and $\mathrm{T}_{3} \times \mathrm{L}_{2}$ for number of kernels/ row; $\mathrm{T}_{3} \times \mathrm{L}_{2}$ for 100-kernel weight. For grain yield/ plant, seven, four and two top crosses at early sowing date; two, three and three top crosses at late sowing date; six, two and three top crosses at the combined analysis for testers $T_{1}, T_{2}$ and $T_{3}$, respectively. The best $\hat{S}_{i j}$ effects were obtained from top crosses $\mathrm{T}_{3} \mathrm{XL}_{2}$ at both and across sowing date. Furthermore, the tester $\mathrm{T}_{3}$ (Sakha Pop.) exhibited the widest range between $\hat{S}_{\mathrm{ij}}$ effects for this trait at the combined analysis. Therefore, the immediate use of lines $\mathrm{L}_{4}, \mathrm{~L}_{5}, \mathrm{~L}_{10}$ and $\mathrm{L}_{12}$ as parents in the development of three way crosses with $\mathrm{T}_{2}$ (S.C El-Hosary 101). Also, the immediate use of lines $\mathrm{L}_{1}, \mathrm{~L}_{2}$ and $\mathrm{L}_{9}$ as parents in development of single crosses with $\mathrm{T}_{1}\left(\mathrm{~L}_{212}\right)$. 
Table 1. Mean squares from ordinary analysis of variance and combining ability for the studied traits.

\begin{tabular}{|c|c|c|c|c|c|c|c|c|c|}
\hline SOV & d.f & $\begin{array}{l}\text { Dayes to } \\
50 \% \text { shed }\end{array}$ & $\begin{array}{l}\text { Dayes to } \\
50 \% \text { silk }\end{array}$ & Plant height & Ear height & $\begin{array}{l}\text { Number of } \\
\text { rows/ ear }\end{array}$ & $\begin{array}{c}\text { Number of kernels/ } \\
\text { row }\end{array}$ & $\begin{array}{c}\text { 100-kernel } \\
\text { weight }\end{array}$ & $\begin{array}{c}\text { Grain yield / } \\
\text { plant }\end{array}$ \\
\hline \multicolumn{10}{|l|}{ Early sowing } \\
\hline Rep & 2 & $9.70 * *$ & $31.25 * *$ & $982.48 * *$ & $330.87 * *$ & $3.73 * *$ & 11.30 & 7.76 & 168.96 \\
\hline Crosses & 44 & $35.15 * *$ & $7.06 * *$ & $453.60 * *$ & $480.52 * *$ & $5.49 * *$ & $29.33 * *$ & $38.55 * *$ & $2198.47 * *$ \\
\hline Lines & 14 & $29.87 * *$ & $11.07 *$ & $692.66 * *$ & $864.04 * *$ & $6.18 * *$ & $52.16^{* *}$ & $37.73 * *$ & $2007.70 * *$ \\
\hline Testers & 2 & $20.55 * *$ & $6.32 * *$ & $4271.99 * *$ & $2626.50 * *$ & 0.33 & $30.84 * *$ & $389.42 * *$ & $10311.58^{* * *}$ \\
\hline Lines $\mathrm{x}$ testers & 28 & $38.84 * *$ & $5.10 * *$ & 61.33 & $135.48 * *$ & $5.50 * *$ & $17.80 * *$ & 13.91 & $1714.35 * *$ \\
\hline Error & 88 & 0.58 & 1.22 & 162.63 & 41.03 & 0.27 & 6.65 & 11.52 & 91.90 \\
\hline GCA & & 0.00 & 0.02 & 4.99 & 4.39 & 0.00 & 0.15 & 0.31 & 6.16 \\
\hline SCA & & 12.75 & 1.30 & 0.00 & 31.48 & 1.74 & 3.72 & 0.80 & 540.82 \\
\hline GCA/SCA & & low & low & low & low & low & low & low & low \\
\hline \multicolumn{10}{|l|}{ late sowing date } \\
\hline Rep & 2 & 0.58 & $8.76^{*}$ & 142.91 & $318.36 * *$ & $2.64 * *$ & 2.30 & 1.09 & 180.61 \\
\hline Crosses & 44 & $66.22 * *$ & $48.19 * *$ & $692.84 * *$ & $311.00 * *$ & $3.84 * *$ & $18.98 * *$ & $47.76 * *$ & $1945.20 * *$ \\
\hline Lines & 14 & $106.60 * *$ & $31.29 * *$ & $1140.67 * *$ & $625.55 * *$ & $5.19 * *$ & $34.41 * *$ & $46.62 * *$ & $2354.32 * *$ \\
\hline $\begin{array}{c}\text { Testers } \\
\end{array}$ & 2 & $160.26^{*}$ & $32.19 * *$ & $5000.46 * *$ & $507.65^{*}$ & $4.08 * *$ & 2.62 & $458.87 * *$ & $6128.08 * *$ \\
\hline Line x Testers x D & & $40.82 * *$ & $57.79 * *$ & $161.23 * *$ & $139.68 * *$ & $3.15 * *$ & $12.43^{*}$ & $18.96 * *$ & $1441.86 * *$ \\
\hline Error & 88 & 1.29 & 2.58 & 85.41 & 48.82 & 0.27 & 8.00 & 7.37 & 118.40 \\
\hline GCA & & 0.32 & 0.00 & 6.77 & 2.18 & 0.01 & 0.08 & 0.37 & 6.41 \\
\hline SCA & & 13.18 & 18.40 & 25.28 & 30.29 & 0.96 & 1.48 & 3.86 & 441.15 \\
\hline GCA/ SCA & & low & low & low & low & low & low & low & low \\
\hline \multicolumn{10}{|c|}{ Combined analysis } \\
\hline Sowing date (D) & 1 & $1978.79 * *$ & $2582.81 * *$ & $31597.39 * *$ & $11446.53 * *$ & $52.56 * *$ & $3828.25 * *$ & $333.33 * *$ & $131288.20 * *$ \\
\hline Rep/D & 4 & $5.14 * *$ & $20.01 * *$ & $562.69 * *$ & $324.62 * *$ & $3.19 * *$ & 6.80 & 4.42 & 174.78 \\
\hline Crosses & 44 & $56.78 * *$ & $27.49 * *$ & $955.95 * *$ & $661.83 * *$ & $8.90 * *$ & $43.42 * *$ & $84.24 * *$ & $3648.14 * *$ \\
\hline Lines & 14 & $98.17 * *$ & $21.90 * *$ & $1493.84 * *$ & $1350.28 * *$ & $11.00 * *$ & $81.82 * *$ & $81.97 * *$ & $4075.07 * *$ \\
\hline Testers & 2 & $66.01 * *$ & $32.58 * *$ & $9258.10 * *$ & $2657.07 * *$ & $3.25 * *$ & 12.76 & $846.34 * *$ & $16055.79 * *$ \\
\hline Lines $\mathrm{x}$ testers & 28 & $35.42 * *$ & $29.92 * *$ & 93.99 & $175.09 * *$ & $8.25 * *$ & $26.41 * *$ & $30.93 * *$ & $2548.40 * *$ \\
\hline Crosses x d & 44 & $44.60 * *$ & $27.76^{* *}$ & $190.49 *$ & $129.69 * *$ & 0.43 & 4.89 & 2.07 & $495.54 * *$ \\
\hline Line $x$ D & 14 & $35.30 * *$ & $20.46^{* *}$ & $339.48 * *$ & $139.31 * *$ & 0.38 & 4.74 & 2.38 & $286.95 * *$ \\
\hline Testers x D & 2 & $114.80 * *$ & $5.93 *$ & 14.35 & $477.08 * *$ & $1.16^{*}$ & $20.70^{*}$ & 1.94 & $383.87 *$ \\
\hline Line $x$ Testers x D & 28 & $44.24 * *$ & $32.97 * *$ & 128.58 & $100.07 * *$ & $0.40 *$ & 3.83 & 1.93 & $607.81 * *$ \\
\hline Error & 176 & 0.93 & 1.90 & 124.02 & 44.93 & 0.27 & 7.32 & 9.45 & 105.15 \\
\hline GCA & & 0.29 & 0.32 & 96.92 & 30.01 & 0.00 & 0.22 & 8.02 & 144.25 \\
\hline
\end{tabular}




\begin{tabular}{|c|c|c|c|c|c|c|c|c|}
\hline $\mathrm{SCA}$ & 000 & 000 & 000 & 1250 & 131 & 376 & 483 & 32343 \\
\hline$\frac{\text { SCA }}{2 C A C A}$ & 0.00 & 0.00 & 0.00 & $\frac{12.50}{\text { high }}$ & $\frac{1.31}{\text { high }}$ & $\frac{3.16}{\text { high }}$ & 4.83 & 323.43 \\
\hline $\begin{array}{l}\text { GCA/SCA } \\
\text { GCA XD }\end{array}$ & $\frac{m g n}{-054}$ & $\frac{m g n}{004}$ & $\frac{\text { mign }}{8982}$ & $\frac{\operatorname{mgn}}{2022}$ & $\frac{\text { mign }}{-005}$ & $\frac{\operatorname{mgn}}{-0266}$ & $\frac{10 \mathrm{~W}}{756}$ & $\frac{10 w}{12120}$ \\
\hline $\begin{array}{l}\text { GCA XD } \\
\text { SCA D }\end{array}$ & $\frac{-0.54}{1444}$ & $\frac{0.04}{1036}$ & $\frac{89.82}{1.52}$ & $\frac{20.22}{1838}$ & $\frac{-0.05}{0.04}$ & -0.200 & $\begin{array}{l}1.50 \\
251\end{array}$ & $\frac{121.20}{16755}$ \\
\hline
\end{tabular}

${ }^{*}$ and $^{* *}$ indicate significance at 0.05 and 0.01 levels of probability, respectively. 
Table 2. Mean performance of the line $\mathrm{x}$ tester crosses and check cultivars in combined across sowing dates.

\begin{tabular}{|c|c|c|c|c|c|c|c|c|c|c|}
\hline \multirow{2}{*}{ Genotype } & \multirow{2}{*}{$\begin{array}{c}\text { Days to } \\
50 \% \\
\text { tasseling } \\
\text { days }\end{array}$} & \multirow{2}{*}{$\begin{array}{c}\text { Days to } 50 \% \text { silking } \\
\text { days }\end{array}$} & \multirow{2}{*}{$\begin{array}{l}\text { Plant height } \\
\quad(\mathrm{cm})\end{array}$} & \multirow{2}{*}{$\begin{array}{l}\text { ear height } \\
\quad(\mathrm{cm})\end{array}$} & \multirow{2}{*}{ No of rows ear ${ }^{-1}$} & \multirow{2}{*}{ No of kernels row ${ }^{-1}$} & \multirow{2}{*}{$\begin{array}{l}\text { 100-kernel weight } \\
\text { (g) }\end{array}$} & \multicolumn{2}{|c|}{ grain yield $(\mathrm{g})$ plant $^{-1}$} & \multirow[b]{2}{*}{ Comb. } \\
\hline & & & & & & & & D1 & D2 & \\
\hline L1x T1 & 54.67 & 59.87 & 222.63 & 105.33 & 12.14 & 33.12 & 32.75 & 143.00 & 101.53 & 122.27 \\
\hline L2xT1 & 56.74 & 60.75 & 230.46 & 112.33 & 10.28 & 29.80 & 34.83 & 119.40 & 95.33 & 107.37 \\
\hline L3 xT1 & 61.42 & 62.00 & 253.61 & 120.33 & 11.39 & 31.77 & 31.58 & 125.47 & 92.53 & 109.00 \\
\hline L4 xT1 & 60.53 & 63.00 & 252.18 & 121.83 & 11.69 & 32.77 & 37.08 & 154.93 & 94.33 & 124.63 \\
\hline L5 xT1 & 57.92 & 58.92 & 257.61 & 126.00 & 10.64 & 31.77 & 37.42 & 126.33 & 93.53 & 109.93 \\
\hline L6 xT1 & 58.79 & 61.67 & 237.64 & 107.00 & 12.75 & 36.78 & 34.67 & 174.67 & 117.18 & 145.93 \\
\hline L7 xT1 & 60.66 & 59.98 & 242.48 & 113.67 & 12.96 & 39.53 & 33.83 & 198.00 & 144.00 & 171.00 \\
\hline L8 xT1 & 59.61 & 61.28 & 243.81 & 109.00 & 11.19 & 37.59 & 35.25 & 155.33 & 104.30 & 129.82 \\
\hline L9 xT1 & 49.79 & 60.00 & 251.43 & 119.50 & 11.34 & 37.50 & 36.00 & 151.67 & 124.94 & 138.30 \\
\hline L10 xT1 & 57.48 & 60.00 & 251.24 & 133.33 & 10.69 & 36.65 & 38.67 & 158.07 & 124.00 & 141.03 \\
\hline L11 xT1 & 58.23 & 59.17 & 260.21 & 134.83 & 12.80 & 35.21 & 39.08 & 167.63 & 140.05 & 153.84 \\
\hline L12 xT1 & 61.60 & 69.44 & 238.41 & 111.67 & 14.81 & 36.38 & 34.00 & 197.87 & 125.53 & 161.70 \\
\hline L13 xT1 & 56.79 & 60.26 & 233.98 & 110.50 & 11.29 & 37.66 & 32.08 & 153.53 & 113.67 & 133.60 \\
\hline L14 xT1 & 57.92 & 60.75 & 255.13 & 132.00 & 12.32 & 38.08 & 38.33 & 191.40 & 154.67 & 173.03 \\
\hline L15 xT1 & 57.55 & 59.75 & 249.06 & 127.50 & 14.29 & 38.16 & 32.58 & 176.93 & 115.08 & 146.01 \\
\hline L1x T2 & 56.54 & 58.04 & 244.41 & 113.00 & 11.52 & 30.55 & 35.83 & 143.37 & 114.17 & 128.77 \\
\hline L2xT2 & 57.52 & 61.50 & 246.11 & 117.33 & 13.91 & 35.73 & 34.00 & 170.07 & 150.67 & 160.37 \\
\hline L3 xT2 & 56.64 & 62.75 & 261.93 & 121.33 & 12.57 & 37.78 & 35.58 & 166.00 & 126.00 & 146.00 \\
\hline L4 xT2 & 60.97 & 63.18 & 257.31 & 126.67 & 12.26 & 33.75 & 34.75 & 148.93 & 113.58 & 131.26 \\
\hline L5 xT2 & 56.79 & 59.56 & 264.12 & 135.00 & 11.41 & 34.66 & 40.00 & 150.87 & 89.13 & 120.00 \\
\hline L6 xT2 & 58.61 & 62.17 & 251.59 & 113.17 & 11.80 & 32.84 & 38.50 & 158.20 & 124.27 & 141.23 \\
\hline L7 xT2 & 57.16 & 59.45 & 246.91 & 122.83 & 12.45 & 39.42 & 43.00 & 188.17 & 135.03 & 161.60 \\
\hline L8 xT2 & 58.54 & 62.36 & 243.20 & 115.50 & 11.83 & 35.18 & 31.92 & 129.57 & 89.20 & 109.38 \\
\hline L9 xT2 & 51.59 & 60.00 & 263.74 & 124.67 & 11.56 & 34.81 & 40.00 & 148.61 & 134.50 & 141.56 \\
\hline L10 xT2 & 57.01 & 59.75 & 254.24 & 137.67 & 11.05 & 38.39 & 43.50 & 188.80 & 114.67 & 151.73 \\
\hline L11 xT2 & 55.52 & 57.70 & 269.88 & 131.33 & 11.96 & 33.72 & 35.58 & 132.27 & 119.77 & 126.02 \\
\hline L12 xT2 & 57.60 & 57.10 & 248.32 & 116.00 & 14.18 & 34.43 & 37.75 & 184.40 & 133.58 & 158.99 \\
\hline L13 xT2 & 50.37 & 58.00 & 248.74 & 131.00 & 11.17 & 39.48 & 35.00 & 148.93 & 126.52 & 137.73 \\
\hline L14 xT2 & 52.83 & 60.00 & 264.74 & 149.17 & 12.31 & 37.81 & 38.58 & 177.67 & 125.30 & 151.48 \\
\hline L15 xT2 & 56.63 & 60.25 & 261.71 & 131.00 & 15.11 & 38.39 & 36.00 & 196.30 & 131.80 & 164.05 \\
\hline L1x T3 & 58.04 & 61.61 & 251.65 & 118.17 & 12.93 & 34.89 & 42.50 & 183.67 & 165.93 & 174.80 \\
\hline L2xT3 & 64.12 & 61.75 & 249.59 & 120.33 & 15.98 & 38.56 & 45.92 & 264.87 & 217.33 & 241.10 \\
\hline
\end{tabular}




\begin{tabular}{|c|c|c|c|c|c|c|c|c|c|c|}
\hline L3 xT3 & 55.59 & 57.25 & 272.38 & 127.33 & 11.59 & 36.07 & 38.92 & 174.00 & 106.53 & 140.27 \\
\hline L4 xT3 & 55.29 & 56.65 & 267.16 & 138.67 & 12.23 & 32.17 & 41.67 & 148.73 & 111.67 & 130.20 \\
\hline L5 xT3 & 59.97 & 61.36 & 269.71 & 143.00 & 12.11 & 32.07 & 44.00 & 176.53 & 121.33 & 148.93 \\
\hline L6 xT3 & 60.15 & 63.86 & 264.60 & 129.17 & 11.32 & 36.53 & 40.33 & 174.93 & 120.92 & 147.93 \\
\hline L7 xT3 & 59.66 & 59.48 & 254.64 & 132.33 & 11.78 & 39.00 & 43.00 & 195.33 & 149.33 & 172.33 \\
\hline L8 xT3 & 60.40 & 60.78 & 268.89 & 125.17 & 12.79 & 34.25 & 34.83 & 169.10 & 131.77 & 150.43 \\
\hline L9 xT3 & 54.64 & 58.50 & 272.57 & 140.17 & 13.06 & 35.86 & 41.92 & 216.40 & 158.00 & 187.20 \\
\hline L10 xT3 & 51.15 & 59.25 & 263.24 & 142.33 & 13.34 & 32.43 & 44.17 & 190.47 & 145.67 & 168.07 \\
\hline L11 xT3 & 57.10 & 59.23 & 276.64 & 130.17 & 11.18 & 36.83 & 41.33 & 189.60 & 114.43 & 152.02 \\
\hline L12 xT3 & 60.04 & 59.95 & 258.48 & 118.17 & 11.45 & 37.73 & 39.33 & 157.53 & 114.83 & 136.18 \\
\hline L13 xT3 & 56.27 & 59.25 & 259.29 & 119.83 & 11.78 & 38.91 & 39.00 & 184.73 & 93.25 & 138.99 \\
\hline L14 xT3 & 52.53 & 60.50 & 278.40 & 137.17 & 11.43 & 39.15 & 40.42 & 184.60 & 142.00 & 163.30 \\
\hline L15 xT3 & 55.55 & 61.25 & 276.88 & 124.17 & 12.88 & 39.50 & 41.50 & 194.60 & 185.00 & 189.80 \\
\hline T.W. 352 & 60.48 & 62.77 & 237.33 & 126.07 & 14.10 & 28.95 & 31.83 & 174.00 & 95.21 & 122.37 \\
\hline SC G 168 & 62.50 & 65.40 & 261.55 & 128.50 & 14.25 & 34.51 & 35.67 & 198.00 & 145.55 & 171.78 \\
\hline L. S. D 5\% & 1.14 & 1.56 & 12.60 & 7.58 & 0.59 & 3.16 & 1.81 & 15.50 & 17.59 & 11.60 \\
\hline L. S. D $1 \%$ & 1.49 & 2.05 & 16.56 & 9.97 & 0.77 & 4.15 & 2.38 & 20.48 & 23.25 & 15.25 \\
\hline
\end{tabular}

D1, D2 and Comb. refer to early, late planting date and combined analysis across planting dates, respectively. 
Table 3. General combining ability effects for all the studied traits.

\begin{tabular}{|c|c|c|c|c|c|c|c|c|c|c|}
\hline & \multirow{2}{*}{$\begin{array}{c}\text { Days to } 50 \% \\
\text { tasseling }\end{array}$} & \multirow{2}{*}{$\begin{array}{l}\text { Days to } 50 \% \\
\text { silking }\end{array}$} & \multirow{2}{*}{$\begin{array}{c}\text { Plant } \\
\text { height }\end{array}$} & \multirow{2}{*}{$\begin{array}{c}\text { Ear } \\
\text { Height }\end{array}$} & \multirow{2}{*}{ No of rows ear ${ }^{-1}$} & \multirow{2}{*}{ No of kernel row ${ }^{-1}$} & \multirow{2}{*}{ 100-kernel weight } & \multicolumn{3}{|c|}{ Grain yield plant $^{-1}$} \\
\hline & & & & & & & & D1 & D2 & Comb. \\
\hline \multicolumn{11}{|l|}{ Tester } \\
\hline 1 & $0.77 * *$ & $0.69 * *$ & $-10.03 * *$ & $-5.83 * *$ & $-0.22 * *$ & -0.34 & $-2.72 * *$ & $-9.97 * *$ & $-9.44 * *$ & $-9.71 * *$ \\
\hline 2 & $-0.92 * *$ & $-0.31 *$ & -0.22 & 0.9 & 0.08 & -0.06 & -0.6 & $-7.45 * *$ & $-3.61 *$ & $-5.53 * *$ \\
\hline 3 & 0.16 & $-0.38 * *$ & $10.25 * *$ & $4.93 * *$ & $0.13 *$ & 0.4 & $3.32 * *$ & $17.42 * *$ & $13.05 * *$ & $15.23 * *$ \\
\hline L.S.D. (gi) $5 \%$ & 0.21 & 0.28 & 2.3 & 1.38 & 0.11 & 0.56 & 0.64 & 2.8 & 3.18 & 2.12 \\
\hline L.S.D. (gi) $1 \%$ & 0.27 & 0.37 & 3.02 & 1.82 & 0.14 & 0.73 & 0.83 & 3.68 & 4.18 & 2.78 \\
\hline L.S.D. (gi-gj) $5 \%$ & 0.29 & 0.4 & 3.25 & 1.96 & 0.15 & 0.79 & 0.9 & 3.96 & 4.5 & 3 \\
\hline L.S.D. (gi-gj) $1 \%$ & 0.39 & 0.53 & 4.28 & 2.57 & 0.2 & 1.04 & 1.18 & 5.21 & 5.91 & 3.94 \\
\hline \multicolumn{11}{|l|}{ Line } \\
\hline 1 & $-0.80 * *$ & -0.59 & $-15.79 * *$ & $-12.65 * *$ & -0.06 & $-3.01 * *$ & -0.91 & $-12.91 * *$ & 1.73 & $-5.59 *$ \\
\hline 2 & $2.25 * *$ & $0.90 * *$ & $-13.30 * *$ & $-8.15 * *$ & $1.13 * *$ & -1.16 & 0.32 & $15.19 * *$ & $28.96 * *$ & $22.07 * *$ \\
\hline 3 & $0.67 * *$ & 0.24 & $7.28 * *$ & -1.81 & $-0.40 * *$ & -0.65 & $-2.57 * *$ & $-14.43 * *$ & $-17.13^{* *}$ & $-15.78 * *$ \\
\hline 4 & $1.72 * *$ & 0.51 & 3.53 & $4.24 * *$ & -0.2 & $-2.96 * *$ & -0.1 & $-18.72 * *$ & $-18.96 * *$ & $-18.84 * *$ \\
\hline 5 & $1.02 * *$ & -0.48 & $8.46 * *$ & $9.85 * *$ & $-0.87 * *$ & $-3.03 * *$ & $2.54 * *$ & $-18.34 * *$ & $-24.15^{* *}$ & $-21.25^{* *}$ \\
\hline 6 & $1.97 * *$ & $2.14 * *$ & -4.08 & $-8.37 * *$ & $-0.30^{*}$ & -0.47 & -0.1 & -0.32 & -4.7 & -2.51 \\
\hline 7 & $1.95 * *$ & $-0.79 *$ & $-7.34 * *$ & -1.87 & 0.14 & $3.46 * *$ & $2.01 * *$ & $24.25 * *$ & $17.30 * *$ & $20.77 * *$ \\
\hline 8 & $2.31 * *$ & $1.04 * *$ & -3.39 & $-8.26 * *$ & $-0.32 * *$ & -0.19 & $-3.93 * *$ & $-18.25 * *$ & $-17.06^{* *}$ & $-17.66^{* *}$ \\
\hline 9 & $-5.21 * *$ & $-0.93 * *$ & $7.23 * *$ & $3.30 *$ & $-0.27 *$ & 0.2 & 1.37 & 2.64 & $13.66^{* *}$ & $8.15^{* *}$ \\
\hline 10 & $-2.00 * *$ & $-0.76^{*}$ & 0.88 & $12.96 * *$ & $-0.56 * *$ & -0.04 & $4.18 * *$ & $9.52 * *$ & 2.63 & $6.07 *$ \\
\hline 11 & -0.26 & $-1.73 * *$ & $13.56^{* *}$ & $7.30 * *$ & $-0.28 *$ & -0.61 & 0.73 & $-6.42^{*}$ & -0.74 & -3.58 \\
\hline 12 & $2.53 * *$ & $1.73^{* *}$ & $-6.95 * *$ & $-9.54 * *$ & $1.23 * *$ & 0.32 & -0.91 & $10.35 * *$ & -0.84 & $4.75^{*}$ \\
\hline 13 & $-2.73 * *$ & $-1.26 * *$ & $-8.02 * *$ & $-4.37 * *$ & $-0.84 * *$ & $2.82 * *$ & $-2.57 * *$ & $-7.19^{*}$ & $-14.34 * *$ & $-10.76^{* *}$ \\
\hline 14 & $-2.78 * *$ & -0.01 & $10.74 * *$ & $14.63 * *$ & -0.23 & $2.49 * *$ & 1.18 & $14.97 * *$ & $15.17 * *$ & $15.07 * *$ \\
\hline 15 & $-0.64 * *$ & -0.01 & $7.20 * *$ & 2.74 & $1.84 * *$ & $2.82 * *$ & -1.24 & $19.69 * *$ & $18.48 * *$ & $19.08 * *$ \\
\hline L.S.D. 5\% (gi) & 0.46 & 0.64 & 5.14 & 3.1 & 0.24 & 1.25 & 1.42 & 6.26 & 7.11 & 4.74 \\
\hline L.S.D. $1 \%$ (gi) & 0.61 & 0.84 & 6.76 & 4.07 & 0.32 & 1.64 & 1.87 & 8.23 & 9.34 & 6.23 \\
\hline L.S.D. 5\% (gi-gj) & 0.66 & 0.9 & 7.28 & 4.38 & 0.34 & 1.77 & 2.01 & 8.86 & 10.05 & 6.7 \\
\hline L.S.D. $1 \%(\mathrm{gi}-\mathrm{gj})$ & 0.86 & 1.18 & 9.56 & 5.76 & 0.45 & 2.32 & 2.64 & 11.64 & 13.21 & 8.81 \\
\hline
\end{tabular}

$*$ and $* *$ indicate significance at 0.05 and 0.01 levels of probability, respectively.

D1, D2 and Comb. refer to early, late planting date and combined analysis across planting dates, respectively. 
Table 4. Specific combining ability effects for all the studied traits.

\begin{tabular}{|c|c|c|c|c|c|c|c|c|c|c|}
\hline \multirow{2}{*}{$\begin{array}{l}\text { top } \\
\text { Crosses }\end{array}$} & \multirow{2}{*}{$\begin{array}{c}\text { Days to } \\
50 \% \\
\text { tasseling }\end{array}$} & \multirow{2}{*}{$\begin{array}{l}\text { Days to } 50 \% \\
\text { silking }\end{array}$} & \multirow{2}{*}{$\begin{array}{l}\text { Plant } \\
\text { height }\end{array}$} & \multirow{2}{*}{$\begin{array}{c}\text { Ear } \\
\text { height }\end{array}$} & \multirow{2}{*}{$\begin{array}{c}\text { No of rows } \\
\text { ear }^{-1}\end{array}$} & \multirow{2}{*}{$\begin{array}{c}\text { No of kernels } \\
\text { row }^{-1}\end{array}$} & \multirow{2}{*}{$\begin{array}{l}\text { 100-kernel } \\
\text { weight }\end{array}$} & \multicolumn{3}{|c|}{ Grain yield plant $^{-1}$} \\
\hline & & & & & & & & D1 & D2 & Comb. \\
\hline T1xL1 & $-2.52 * *$ & -0.66 & -6.9 & -1.01 & 0.16 & 0.61 & -1.56 & -3.7 & $16.24 * *$ & $-9.97 *$ \\
\hline T1xL2 & $-3.49 * *$ & $-1.28 *$ & -1.56 & 1.49 & $-2.89 * *$ & $-4.56^{* *}$ & -0.69 & $\begin{array}{c}- \\
55.40 * * \\
\end{array}$ & $\begin{array}{c}- \\
49.67 * * \\
\end{array}$ & $\begin{array}{c}- \\
52.54 * * \\
\end{array}$ \\
\hline T1xL3 & $2.77 * *$ & 0.64 & 1 & 3.16 & -0.24 & $-3.10 * *$ & -1.06 & $\begin{array}{c}- \\
19.72 * *\end{array}$ & -6.38 & $13.05^{* *}$ \\
\hline T1xL4 & $0.84 *$ & $1.36 *$ & 3.32 & -1.4 & -0.15 & 0.21 & 1.97 & $14.04 *$ & -2.75 & 5.64 \\
\hline T1xL5 & $-1.07 * *$ & $-1.72 * *$ & 3.82 & -2.84 & $-0.53 *$ & -0.72 & -0.33 & $14 . \overline{-} * *$ & 1.64 & -6.65 \\
\hline T1xL6 & $-1.16^{* *}$ & $-1.59 * *$ & -3.61 & -3.62 & $1.01 * *$ & 1.74 & -0.44 & $15.37 * *$ & 5.83 & $10.60 *$ \\
\hline T1xL7 & 0.73 & -0.35 & 4.5 & -3.45 & $0.78 * *$ & 0.56 & $-3.39 * *$ & $14.14 *$ & 10.65 & $12.40 * *$ \\
\hline T1xL8 & -0.68 & -0.89 & 1.88 & -1.73 & $-0.52 *$ & $2.26^{*}$ & $3.97 * *$ & $13.97 *$ & 5.32 & $9.65 *$ \\
\hline T1xL9 & $-2.99 * *$ & -0.19 & -1.12 & -2.79 & $-0.43^{*}$ & 1.79 & -0.58 & -10.59 & -4.77 & -7.68 \\
\hline T1xL10 & $1.50 * *$ & -0.36 & 5.03 & 1.38 & $-0.78 * *$ & 1.17 & -0.72 & $-11.07 *$ & 5.33 & -2.87 \\
\hline T1xL11 & 0.51 & -0.22 & 1.33 & $8.55^{* *}$ & $1.04 * *$ & 0.3 & $3.14 *$ & $14.44 * *$ & $24.74 * *$ & $19.59 * *$ \\
\hline T1xL12 & $1.09 * *$ & $6.58 * *$ & 0.03 & 2.21 & $1.55 * *$ & 0.54 & -0.31 & $27.91 * *$ & 10.32 & $19.11 * *$ \\
\hline T1xL13 & $1.55^{* *}$ & 0.4 & -3.33 & -4.12 & 0.09 & -0.68 & -0.56 & 1.11 & 11.96 & 6.53 \\
\hline T1xL14 & $2.73 * *$ & -0.36 & -0.93 & -1.62 & $0.52 *$ & 0.07 & 1.94 & $16.82 * *$ & $23.45 * *$ & $20.13 * *$ \\
\hline T1xL15 & 0.21 & $-1.36^{*}$ & -3.46 & $5.77 *$ & 0.41 & -0.18 & -1.39 & -2.37 & $\begin{array}{c}- \\
19.44 * * \\
\end{array}$ & $\begin{array}{c}- \\
10.91 * * \\
\end{array}$ \\
\hline T2xL1 & $1.05^{*}$ & $-1.49 * *$ & 5.07 & -0.06 & $-0.76 * *$ & $-2.24 *$ & -0.59 & -5.87 & -9.44 & -7.65 \\
\hline T2xL2 & $-1.02 *$ & 0.48 & 4.28 & -0.23 & $0.44 *$ & 1.1 & $-3.65 * *$ & -7.27 & -0.17 & -3.72 \\
\hline T2xL3 & -0.32 & $2.39 * *$ & -0.49 & -2.56 & $0.64 * *$ & $2.64 *$ & 0.82 & $18.29 * *$ & $21.25 * *$ & $19.77 * *$ \\
\hline T2xL4 & $2.96 * *$ & $2.55 * *$ & -1.35 & -3.29 & 0.12 & 0.92 & $-2.48 *$ & 5.51 & 10.66 & 8.09 \\
\hline T2xL5 & -0.52 & -0.08 & 0.53 & -0.56 & -0.06 & 1.89 & 0.13 & 7.07 & -8.59 & -0.76 \\
\hline T2xL6 & 0.35 & -0.09 & 0.54 & -4.17 & -0.24 & $-2.48 *$ & 1.27 & -3.62 & 7.08 & 1.73 \\
\hline T2xL7 & $-1.08 * *$ & 0.12 & -0.88 & -1.01 & -0.03 & 0.16 & $3.66^{*}$ & 1.78 & -4.15 & -1.18 \\
\hline T2xL8 & -0.05 & $1.20 *$ & $-8.54 *$ & -1.95 & -0.19 & -0.43 & -1.48 & $\begin{array}{c}- \\
14.32^{-} * * \\
\end{array}$ & $-15.62 *$ & $\begin{array}{c}- \\
14.97 * * \\
\end{array}$ \\
\hline T2xL9 & 0.51 & 0.81 & 1.38 & -4.34 & $-0.51 *$ & -1.18 & 1.29 & $16.17 * *$ & -1.04 & $-8.60 *$ \\
\hline T2xL10 & $2.72 * *$ & 0.39 & -1.78 & -1.01 & $-0.73 * *$ & $2.63 *$ & 1.99 & $17.13 * *$ & -9.84 & 3.65 \\
\hline
\end{tabular}




\begin{tabular}{|c|c|c|c|c|c|c|c|c|c|c|}
\hline T2xL11 & -0.51 & -0.69 & 1.19 & -1.67 & -0.1 & -1.47 & $-2.48 *$ & $23.45^{-} *$ & -1.38 & $12 . \overline{4} 2^{*} *$ \\
\hline T2xL12 & $-1.22 * *$ & $-4.75 * *$ & 0.15 & -0.17 & $0.62 * *$ & -1.68 & 1.32 & $11.91 *$ & $12.54 *$ & $12.23 * *$ \\
\hline T2xL13 & $-3.18 * *$ & -0.86 & 1.63 & $9.66 * *$ & -0.33 & 0.86 & 0.24 & -6.02 & $18.98 * *$ & 6.48 \\
\hline T2xL14 & -0.68 & -0.11 & -1.13 & $8.83 * *$ & 0.21 & -0.48 & 0.07 & 0.56 & -11.75 & -5.6 \\
\hline T2xL15 & $0.98^{*}$ & 0.14 & -0.61 & 2.55 & $0.93 * *$ & -0.23 & -0.09 & $14.47 * *$ & -8.55 & 2.96 \\
\hline T3xL1 & $1.47 * *$ & $2.15 * *$ & 1.83 & 1.07 & $0.60 * *$ & 1.64 & 2.15 & 9.57 & $25.67 * *$ & $17.62 * *$ \\
\hline T3xL2 & $4.51 * *$ & 0.8 & -2.72 & -1.26 & $2.45 * *$ & $3.46 * *$ & $4.34 * *$ & $62.67 * *$ & $49.84 * *$ & $56.26^{* *}$ \\
\hline T3xL3 & $-2.45 * *$ & $-3.03 * *$ & -0.51 & -0.6 & -0.39 & 0.46 & 0.23 & 1.43 & $-14.87 *$ & -6.72 \\
\hline T3xL4 & $-3.80 * *$ & $-3.91 * *$ & -1.97 & 4.68 & 0.03 & -1.13 & 0.51 & $\begin{array}{c}- \\
19.55^{* *}\end{array}$ & -7.91 & $\begin{array}{c}- \\
13.73 * *\end{array}$ \\
\hline T3xL5 & $1.59 * *$ & $1.80 * *$ & -4.35 & 3.4 & $0.59 * *$ & -1.17 & 0.21 & 7.87 & 6.95 & 7.41 \\
\hline T3xL6 & $0.81 *$ & $1.68^{* *}$ & 3.07 & $7.79 * *$ & $-0.77 * *$ & 0.74 & -0.82 & $-11.75^{*}$ & $-12.92 *$ & $12 . \overline{-}+* *$ \\
\hline T3xL7 & 0.34 & 0.23 & -3.62 & 4.46 & $-0.75^{* *}$ & -0.72 & -0.27 & $15.92 * *$ & -6.5 & $\begin{array}{c}- \\
11.21 * * \\
\end{array}$ \\
\hline T3xL8 & 0.73 & -0.31 & 6.67 & 3.68 & $0.72 * *$ & -1.83 & $-2.49 *$ & 0.35 & 10.3 & 5.32 \\
\hline T3xL9 & $2.48 * *$ & -0.62 & -0.26 & $7.13 * *$ & $0.94 * *$ & -0.6 & -0.71 & $26.76^{* *}$ & 5.81 & $16.28 * *$ \\
\hline T3xL10 & $-4.22 * *$ & -0.03 & -3.25 & -0.37 & $1.51 * *$ & $-3.80 * *$ & -1.27 & -6.06 & 4.51 & -0.78 \\
\hline T3xL11 & 0 & 0.91 & -2.52 & $-6.87 *$ & $-0.93 * *$ & 1.17 & -0.66 & 9.01 & $\begin{array}{c}- \\
23.36 * * \\
\end{array}$ & -7.17 \\
\hline T3xL12 & 0.14 & $-1.83 * *$ & -0.18 & -2.04 & $-2.16^{* *}$ & 1.15 & -1.02 & $\begin{array}{c}- \\
39.82 * * \\
\end{array}$ & $\begin{array}{c}- \\
22.86 * * \\
\end{array}$ & $\begin{array}{c}- \\
31.34 * * \\
\end{array}$ \\
\hline T3xL13 & $1.63 * *$ & 0.46 & 1.7 & $-5.54 *$ & 0.23 & -0.18 & 0.32 & 4.91 & $\begin{array}{c}- \\
30.94 * * \\
\end{array}$ & $13.01 * *$ \\
\hline T3xL14 & $-2.05 * *$ & 0.47 & 2.06 & $-7.21 * *$ & $-0.72 * *$ & 0.4 & -2.02 & $\begin{array}{c}- \\
17.37 * * \\
\end{array}$ & -11.7 & $\begin{array}{c}- \\
14.54 * * \\
\end{array}$ \\
\hline T3xL15 & $-1.18 * *$ & $1.22 *$ & 4.07 & $-8.32 * *$ & $-1.35 * *$ & 0.41 & 1.48 & $-12.10^{*}$ & $27.99 * *$ & 7.95 \\
\hline L.S.D. (Sij) 5\% & 0.21 & 1.1 & 8.41 & 5.36 & 0.42 & 2.17 & 2.46 & 10.85 & 12.31 & 8.21 \\
\hline $1 \%$ & 0.27 & 1.45 & 11.71 & 7.05 & 0.55 & 2.85 & 3.23 & 14.26 & 16.18 & 10.78 \\
\hline $\begin{array}{l}\text { L.S.D. (Sij- } \\
\text { Ski)5\% }\end{array}$ & 0.29 & 1.56 & 12.6 & 7.58 & 0.59 & 3.06 & 3.48 & 15.34 & 17.41 & 11.6 \\
\hline $1 \%$ & 0.39 & 2.05 & 16.56 & 9.97 & 0.77 & 4.03 & 4.57 & 20.16 & 22.89 & 15.25 \\
\hline
\end{tabular}

${ }^{*}$ and ${ }^{* *}$ indicate significance at 0.05 and 0.01 levels of probability, respectively.

D1, D2 and Comb. refer to early, late planting date and combined analysis across planting dates, respectively. 
From the present study, it could be concluded that testers of broad genetic base are more efficient than those of narrow genetic base for evaluation of $\hat{g}_{i}$ effects for inbred lines of maize

\section{References}

Abd El-Aal A.M.M. (2012). Utilization of line $x$ tester model for evaluating the performance of some new yellow maize inbred lines. Bull. Fac., Agric., Cairo Univ. 63: 29-36.

AL-Naggar, A.M.; H.Y. El-Sherbieny and A.A. Mahmoud (1997): Effectiveness of inbreds, single crosses and populations as testers for combining ability in maize. Egypt. J. Plant Breed. 1: $35-46$

Amer, E.A. (2002). Combining ability on early maturing inbred lines of maize. Egypt. J. Appl. Sci. 42(2): 162:181.

Basbag, S., R. Ekinci and O. Gencer (2007). Combining ability and heterosis for earliness characters in line $\mathrm{x}$ tester population of Gossypium hirsutum L. Hereditas 144:185-190.

Bello, O.B. and G. Olaoye (2009). Combining ability for maize grain yield and other agronomic characters in a typical southern guinea savanna ecology of Nigeria. Afr. J. Biotechnol. 8: 25182522.

Davis, R.L. (1927): Report of the plant breeder.Rep. Puerto Rico. Agric. Exp. Sta. P: 14-15.

El-Badawy, M.EL.M., S.A. Sedhom, A.M. Morsy and A.A.A. El-Hosary, (2010). Combining ability in maize (Zea mays L.) under two nitrogen rates and genetic distance determined by RAPD markers. Proceedings of the $12^{\text {th }}$ International Conference of Agronomy, September 20-22, 2010, EL-Arish, Sinai, Egypt, pp: 48-66.

El-Hosary A.A. (1985). Study of combining ability in some top crosses in maize. Egypt. J. Agron. 10(12) 39-47.

EL-Hosary A.A.A. and A. A. Elgammaal (2013). Utilization of line $\mathrm{x}$ tester model for evaluating the combining ability of some new white maize inbred lines. Egypt. J. Plant Breed. 17(1):79-72.

El-Hosary, A.A. and M.EL.M. El-Badawy, (2005). Heterosis and combining ability in yellow corn (Zea mays L.) under two nitrogen levels. Proceedings of the $11^{\text {th }}$ Conference of Agronomy, November 15-16, 2005, Assiut University, Egypt, pp: 89-99.

El-Hosary, A.A., M.EL.M. El-Badawy and Y.M. Abdel-Tawab (2006). Genetic distance of inbred lines and prediction of maize single-cross performance using RAPD and SSR markers. Egypt. J. Genet. Cytol. 35: 209-224.

El-Hosary, A.A.A. (2014). Relative values of three different testers in evaluating combining ability of new maize inbred lines. International $\mathbf{J}$. of Plant Breeding and genetics 5(2): 57-65.

Gilbert, N.E.G. (1958). Diallel cross in plant breeding. Heredity 12: 477-492.

Hani A. Eltelib, Muna A. Hamad and Eltom E. Ali (2006). The effect of nitrogen and phosphorus fertilization on growth, yield and quality of forage maize (Zea mays L.). Journal of Agronomy 5: 515-518.

Hefny, M.M. and A.A. Aly (2008). Yielding ability and nitrogen use efficiency in maize inbred lines and their crosses. International Journal of Agricultural Research 3: 27-39.

Ibrahim, M.H.A. and M.A. Ghonemy (2010). Evaluation of some new maize inbred lines for combining ability using top cross method. Egypt. J. Plant Breed. 14(1): 217:228.

Jenkins, M.T. (1935): The effect of inbreeding and selection within inbred lines of maize upon the hybrids made after successive generations of selfing. Lowa State J. Sci. 3: 429-450.

Kang, M.S. (1998). Using genotype by environment interaction for crop cultivar development. Adv. Agron., 62:199-252.

Kempthorne, O. (1957). An Introduction to Genetic Statistics. John Wiley and Sons Inc., Landon, New York.

Matzinger D.F. (1953). Comparison of three types of testers for the evaluation of inbred lines of corn. Agron. J. 45:493-495.

Ngaboyisonga, C., K. Njoroge, D. Kirubi and S.M. Githiri (2009). Effects of low nitrogen and drought on genetic parameters of grain yield and endosperm hardness of quality protein maize. Asian J. Agric. Res. 3: 1-10.

Sofi, P. and A.G. Rather (2006). Genetic analysis of yield traits in local and CIMMYT inbred line crosses using line $\mathrm{x}$ tester analysis in maize (Zea mays L.) Asian J. Plant Sci., 5: 1039-1042.

Sprague G.F. and Tatum L.A. (1942). General vs. specific combining ability in single crosses of corn. J. Am. Soc., Agron. 34:923-932.

Sprague, G.F. (1939). An estimation of the number of top crossed plants required for adequate representation of corn variety. J. Am. Soc. Agron. 38: 11-16.

Steel, R.G. and J.H. Torrie (1980). Principles and procedures of statistics. McGraw-Hill Book Company, New York, Toronto, London.

Tamilarasi, P.M. and M. Vetriventhan (2009). Exploitation of promising maize (Zea mays L.) hybrids for nitrogen (N) stress environment by studying the SCA, heterosis and nature of gene action at different $\mathrm{N}$ fertilizer doses. Int. J. Plant Sci. 4: 15-19. 


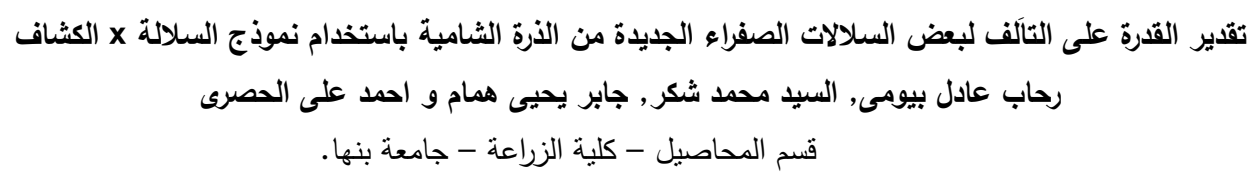

تم تقييم 45 هجين قمى ناتجين من تهجين 15 سلالة 3 x كثافات مع اثثين من الهجن التجارية للمقارنة (هجين فردى 168 و هجين ثلاثى 352) فى تصميم القطاعات الكاملة العشوائية تحت ميعادى زراعة (15 مايو و 15 يونيه) موسم 2017 فى مزرعة كلية الزراعة - جامعة بنها لصفات عدد الايام حتى ظهور 50\% من الحراير , ارتفاع كلا من النبات و الكوز , المحصول و مكوناته. و كانت اهم النتائج: - كان التباين الراجع الى الكثافات و السلالات و التفاعل بين السلالات x الكثافات معنوى فى كلا الميعادين و التحليل المشترك لمعظم الصفات تحت الدراسة. - كان التباين الراجع الى التفاعل بين الهجن القمية و السلالات و الكثافات و السلالات x الكثافات مع ميعادى الزراعة معنويا لمعظم الصفات تحت الدراسة.

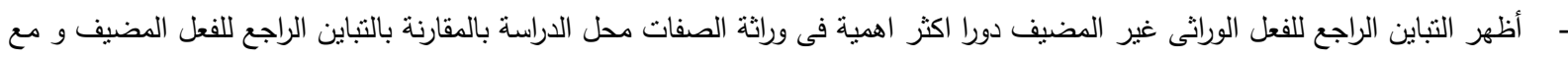
ذللك كان التفاعل بين التأثير غير المضيف X ميعادى الزراعة اعلى من التفاعل بين التأثير المضيف X ميعادى الزراعة لجميع هذه الصفات.

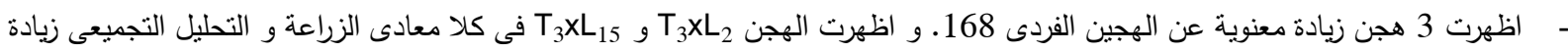
معنوية عن افضل صنف المقارنة (هـ ف 168 ) . و ايضا يوجد 10 هجن قمية لم تظهر معنوية عن افضل صنف مقادئ مقارنة لصفة محصول الحبوب / نبات - اظهرت السلالات2, 7, 14 و 15 قدره عامة على التآلف لصفة محصول حبوب النبات الفردى و السلالات 9, 10 و 13 لصفة التبكير فى التزهير

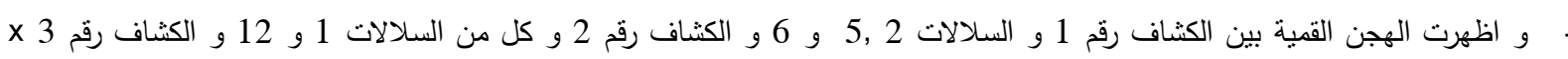

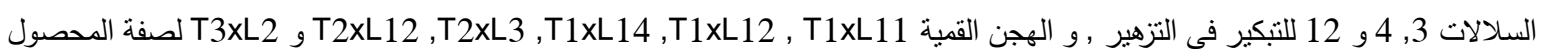
افضل الهجن فى القدرة الخاصة على التآلف. 